\title{
THE COMPARATIVE ANALYSIS OF SCIENTIFIC AND SOCIAL REPRESENTATIONS OF CONSCIOUSNESS AND THE UNCONSCIOUS
}

\author{
Elena V. Levchenko, \\ Anastasia G. Prodovikova \\ Perm State University \\ Perm
}

\begin{abstract}
The article deals with the research into social notions of such integral psychic phenomena as consciousness and the unconscious. The concept of social notions, proposed by S. Moscovici in 1961, was used. This research was carried out in several stages. Firstly, scientific definitions of consciousness and the unconscious were analyzed. Secondly, dictionaries of the Russian language were studied. Then an empirical study of social notions of consciousness and the unconscious was conducted. As many as 240 students of Perm State University took part in the study as subjects. The core-periphery structure of scientific and social representations of consciousness and the unconscious that is typical of modern Russian culture and common to such of its carriers as active researchers in psychology and students was reconstructed. Finally, notions of consciousness were compared to the notions of the unconscious. Studying the notions of psychic phenomena makes it possible to reconstruct the idea of the psychic that is typical of non-professionals. The process of constructing social notions can be explained by the "dynamic model" proposed in the study by S. Moscovici and M. Hewstone (1984).
\end{abstract}

Keywords: social representations, scientific representations, consciousness, the unconscious, "dynamic model" of S. Moscovici and M. Hewstone, the core-periphery structure of scientific and social representations, stages of objectivation.

According to many today's researchers, the concept of social representations, proposed by S. Moscovici in 1961, has made a significant contribution to the development of social psychology (Emelyanova, 2001; Brushlinsky, 1998; Shikhirev, 1999; etc.). The number of studies undertaken in the frames of this approach grows every year, so does the range of issues. As such, various phenomena may be the object of representation: from psychoanalysis (Moscovici, 1961) to democracy, political leadership, and social responsibility (Emelyanova, 2006). 
The subject of our research is the social representations of such integral psychic phenomena as consciousness and the unconscious. The choice of the above subject is not incidental. During the past decades, the number of studies concerned with psychics and psychological phenomena by non-psychologists has notably increased. For example, the following subjects have been studied: children's representations of the soul (Subbotsky, 1985; Savina, 1995), implicit personality theories (Kruglanski, 2007), implicit theories of intelligence (Parkhomenko, 2003), mental representations of emotions (Podprugina, 2003), the "theory of mind" has been developed (Carroll, Apperly, \& Riggs, 2007; Sobel, 2007).

The choice of consciousness and the unconscious as an object of representations was conditioned by the following reasons. First of all, the idea of consciousness has been systematically developed in psychology, beginning with its set-up and separation as an independent branch of knowledge. The idea of the unconscious came to be studied by psychologists later. The introduction of the term "the unconscious" as a scientific category is associated with the works of S. Freud. Besides, the Russian culture has featured an absolutely special approach to psychoanalysis. As such, due to ideological reasons, starting from the 1930s, psychoanalysis and consequently the very concept of "the unconscious" were banned, and free discussion and evaluation of works by S. Freud and his followers became possible only in the 1990s (Levchenko, 2008). Therefore, the idea of consciousness actively developing from the mid $19^{\text {th }}$ century both in domestic and foreign psychology, will for the purpose of this analysis be referred to as a "relatively old" concept. The idea of the unconscious, which appeared in science later and remained prohibited in the Russian psychology of the Soviet period (which stood in the way of its systematic development), will be referred to as a "relatively young" concept. It should be noted that the "age" of idea is estimated with reference to its existence in a certain socio-cultural context, primarily, in the Russian one. Secondly, consciousness and the unconscious are in a fundamental opposition to one another, which simplifies the process of comparison of representations of these phenomena.

Therefore, the present study aims to identify the social representations of consciousness and the unconscious available today in Russia (as exemplified by the representations formed among the students) and describe the external process of their construction, i.e. changes facing the 
aggregate of the given scientific ideas after they become a common sense property.

This research was carried out in several stages. During stage one, scientific definitions of consciousness and the unconscious were selected. These definitions were analyzed during stage two. Russian dictionaries were studied during stage three. Stage four focused on the empirical study of social representations of consciousness and the unconscious. At stage five, findings of the empirical study were processed using the qualitative and quantitative analysis methods. During stage six, a coreperiphery system of scientific and social representations of consciousness and the unconscious was built. Further, at stage seven, we analyzed social representations of consciousness and the unconscious with reference to the phases undergone by a scientific theory in the course of its transformation into a social representation. And finally, representations of consciousness were compared to the representations of the unconscious.

Let us consider the above mentioned stages in a more detailed way. The first and second stages were set to identify the combination of scientific ideas of consciousness and the unconscious. During the first stage, proceeding from the reviews concerned with the issues of consciousness (Akopov, 2004; 2007) and the unconscious (Asmolov, 2002) and also from the analysis of the chapters devoted to the theoretical study of these phenomena in a number of dissertations (Vakhrusheva, 2003; Sitnikov, 2003; Shevchenko, 2003), we compiled a list of recognized leading researchers in the field. Some of these scientists' works in the field, as well as psychological dictionaries, provided definitions of the examined phenomena. The following criteria were used to select the texts containing a definition: 1 . The sentence (or several sentences) must include phrases like "consciousness (the unconscious) is understood as ...," "consciousness (the unconscious) is ...", "consciousness (the unconscious) is regarded as ..., etc.; 2. The paragraph must constitute the author's complete idea; 3 . If more than one definition of consciousness (the unconscious) has been given by the same author, we opted for the definition that has been quoted in works more frequently (reviewing the definitions of consciousness (the unconscious)). During the second stage, we carried out the content analysis of definitions of each phenomenon. A segmental, issue-related count of references to the categories was used, according to which only the first appearance of 
a category in the unit of context was registered, whereas any repeated references to the category in the given unit of context were disregarded. The scale of categories was not set initially and was developed in accordance with the content of the selected definitions. A word or syntactically indivisible collocation served as an indicator of category. The distribution of empirical indicators among categories was controlled by the expert evaluation method.

A survey of scientific psychological literature dealing with the issue of consciousness revealed the traditional for the psychological knowledge polysemy of this notion. During the first stage, having analyzed a number of monographs by some researchers of consciousness (Agafonov, 2003; Allakhverdov, 2000; Bekhterev, 1999; Ganzen, 1984; James, 2001; Jaspers, 2001; Leontiev, 1975; Lomov, 1984; Platonov, 1972; Rubinstein, 1957; Wundt, 2002; Zinchenko, 2001; etc.) and several psychological dictionaries (Meshcheriakov \& Zinchenko, 2003; Reber, 2000; Davydov et al., 1983; etc.), we came to a conclusion that while some authors distinctly articulate the definition of consciousness (Bekhterev, 1999; Zinchenko, 2001; Leontiev, 1975; etc.), others seem to avoid such clarity in every possible way (Vasilyuk, 2007; Luria, 1979; Subbotsky, 1999). For the second stage of analysis, a total of 41 texts containing distinctly articulated definitions were selected based on the criteria specified above (37 definitions given by the authors +4 dictionary clauses). It should be noted that the selected definitions of consciousness cover the time period of 1888 (Bekhterev, 1999) to 2009 (Abulkhanova, 2009). Out of the above mentioned texts, 32 belong to domestic scientists and 9 are the products by foreign authors.

Upon the completion of content analysis of the selected texts, the following ideas were identified as more frequently used in the definition of consciousness: 1) consciousness as reflection, as learning; 2) consciousness as an attitude and experience; 3 ) consciousness as regulation; 4) consciousness as awareness (including self-awareness); 5) consciousness as an integration of inner mental life; 6) consciousness as the top level of mental evolution associated with historical and cultural origin and related to speech; 7) consciousness as a function of something; 8) consciousness as part of psychics. Apart from that, a series of rarely encountered ideas were identified. Those, for instance, included such ideas as "philosophical category" (Meshcheriakov \& Zinchenko, 2003), "accompaniment and provision of a personality's existence with consciousness" 
(Abulkhanova, 2009), "those aspects of mental life that attract a person's attention" (Reber, 2000), "occurs in the morning upon awakening from sleeping without dreams and lasts the whole day until falling asleep again or until you develop a condition, which might be called 'unconscious,' e.g., a coma or death" (Searle, 1980), and some others.

Let us pass on to the representations of the unconscious. The difficulty that we faced in the course of studying scientific literature was the limited number of definitions of this term that was found (the total of 17). The time during which the texts containing the selected definitions were published, covered the period of 1921 to 2007. During the content analysis, all definitions were split into negative and positive. The above division dates back to the old times in the history of psychology. D.N. Uznadze stated that the definition of the unconscious in psychoanalysis had a negative nature and proposed the concept of "set" as its positive definition (as cited in Asmolov, 2002, pp. 31-32). Negative definitions of the unconscious were associated with the denial of characteristics of consciousness (apprehendability, controllability, etc.). The latter group included the overwhelming majority of the analyzed texts (over $80 \%)$. As this kind of research did not make it possible to reconstruct the profound characteristic of the unconscious, we also analyzed the psychological concepts of the examined phenomenon, in which the essence of the given phenomenon was disclosed through the description of its content (Agafonov, 2003; Asmolov, 2002; Freud, 2006; Grof, 2001; Herbart (cited in Zhdan, 2002); Jung, 1994; Leibniz, 1982; Simonov, 2001; Uznadze, 1966). The above analysis helped identify the phenomena that in the author's mind belonged to the area of the unconscious, thus making up for the lack of full-fledged definitions or their undue laconism, like: strong feelings and desires disapproved by the society; low intensity perceptions; memory storage; primitive needs and instincts; archetypes; dream; non-conscious regulators of action performance; reflexes; nonconscious components of creative activity; birth-associated memories; mystical feelings.

Then, from studying the aggregate of scientific ideas related to consciousness and the unconscious, we went on to examine the corresponding social representations. During the third stage, the central core elements of social representations were identified. This was done by studying the details presented in modern definition dictionaries and associative dictionaries of the Russian language. Thus, the S.I. Ozhegov's 
dictionary (Ozhegov, 1989) offers the following interpretation of "consciousness":

1. An ability to reproduce reality in thinking; a psychic activity as a reflection of reality.

2. A condition of an individual in healthy mind and memory, an ability to be aware of his behaviour and feelings.

3. A thought, a feeling, a clear understanding of something.

4. The same as responsibility: an ability to properly sort out reality and identify one's behavior (Ozhegov, 1989).

The term "the unconscious" in Russian does not have the status of a noun and is used as an adjective. Modern definition dictionaries (Ozhegov, 1989; The Russian definition dictionary supplied with etymological data, 2007) provide the following meanings of this word:

1. Accompanied by the loss of consciousness;

2. instinctive, unaccountable.

Let us compare the lexical meanings of the words "consciousness" and "the unconscious" with the scientific ideas of consciousness and the unconscious. By comparing the scientific ideas of "consciousness" with the lexical meanings of this word, we noticed both similarities and differences. The similarity was found with regard to the following ideas: 1) consciousness as reflection and learning; 2) consciousness as awareness (including selfawareness). The dictionary gives two meanings: "a condition of an individual in healthy mind and memory" and "clear understanding of something" which we did not find in scientific definitions of consciousness.

The lexical meanings of the word "unconscious" comply with the scientific ideas of the unconscious identified in the course of the text analysis. The meanings presented in the definition dictionaries belong to the group of "negative definitions" and show similarity with the following scientific ideas of the unconscious: "something that is not apprehended," "something that is not controlled."

During the forth stage, an empirical study of the social representations of consciousness and the unconscious was conducted. This study was assisted by first, second and third year students from various faculties of Perm State University: a total of 240 students ranging from 17 to 22 years old. The number of male and female subjects was about the same. The subjects were not supposed to specialize in psychology and philosophy. The study took place before the students were exposed to a course in general psychology. 
As this work was performed in the traditions of S. Moscovici's school, the qualitative research methodology was selected for the study. Data collection methods included an association experiment, the drawing method and the method of definition. Beside the representations of consciousness and the unconscious, the students' representations of the soul and psychics were studied as well. Introducing the latter in the study enabled us to regard the representations of consciousness and the unconscious not in isolation but in the context of integral concepts used to display the psychic. In other words, we made an attempt to look at "consciousness" and "the unconscious" from the perspective of the idea of the psychic the subjects had.

The fifth stage included the processing of the data obtained during the empirical study. The data on the association experiment were processed with the use of both the prototypical analysis of P. Verges (Bovina \& Dragulskaya, 2006) and the content analysis. The first technique makes it possible to reconstruct the core-periphery structure of social representations. The second method of data processing is a qualitative and quantitative method identifying the key concepts (ideas) and calculating the frequency of their use. Content analysis was also used to process the drawings and definitions obtained from the subjects. We developed the systems of categories of content-and-analytical study of the empirical material, common for the representations of consciousness, the unconscious, the soul and psychics.

As a result of the analysis, we identified an aggregate of the ideas characteristic of social representations of consciousness and the unconscious. The next stage of our study consisted in building the coreperiphery structure of representations. As noted earlier, prototypical analysis is designed specifically for this purpose. As far as the data of content analysis of associations, drawings and definitions is concerned, a procedure similar to prototypical analysis was applied to these data. The same procedure was applied to the results of content analysis of scientific definitions. The key point of this procedure, which was borrowed from P. Verges' prototypical analysis, is that the criterion for the identification of the central core, the potential change area and the peripheral system itself is the frequency of the element, in our case, the category of content analysis. The resulting core-periphery structure of the scientific and social representations is shown in Table 1. The graphic image of the findings is shown in Figures 1 and 2. 


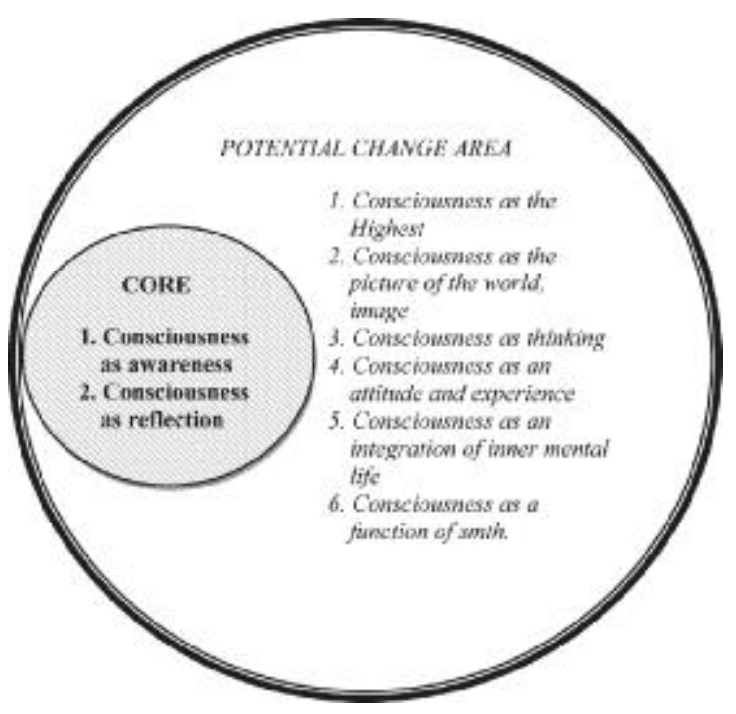

Figure 1. Scientific representations of consciousness

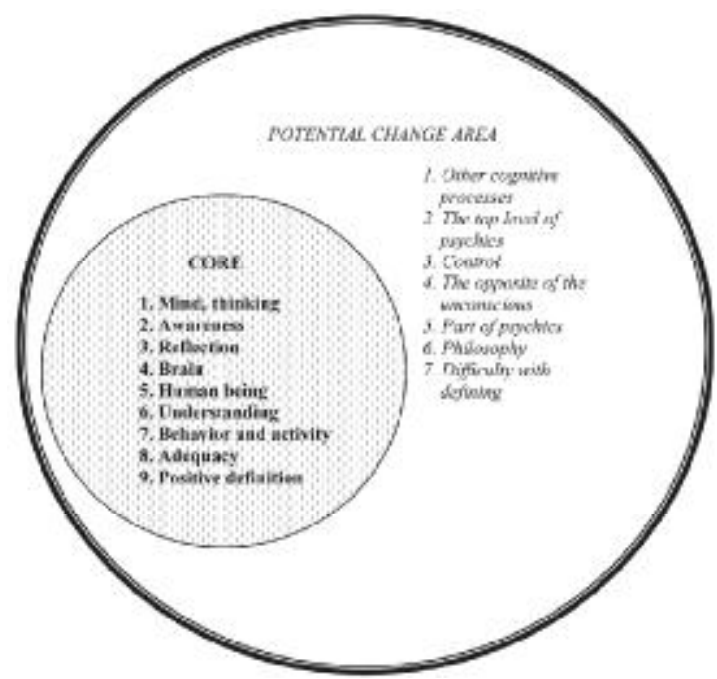

Figure 2. Social representations of consciousness 


\begin{tabular}{|c|c|c|c|c|c|c|c|c|c|c|c|c|c|c|}
\hline 52 & & & & ena & Le & 0, & A & $C$ & Prod & Kova & & & & \\
\hline$\frac{\overrightarrow{0}}{\frac{\pi}{0}}$ & & 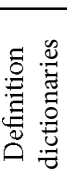 & & 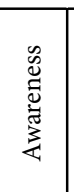 & 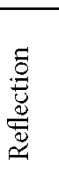 & \begin{tabular}{|l}
$\mid \vec{v}$ \\
芯 \\
离
\end{tabular} & 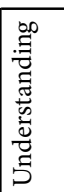 & 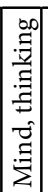 & & & & & & \\
\hline & & है & $\circ$ & \begin{tabular}{l} 
\\
\multirow{1}{*}{}
\end{tabular} & 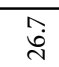 & & & & $\stackrel{\infty}{\infty}$ & $\underline{\underline{S}}$ & \begin{tabular}{|l|} 
\\
n.
\end{tabular} & $\begin{array}{l}0 \\
10\end{array}$ & \begin{tabular}{|l|}
$\infty$ \\
\\
\end{tabular} & \\
\hline $\begin{array}{l}\overline{0} \\
\overline{0} \\
\overline{0} \\
0 \\
0 \\
0 \\
0 \\
0 \\
0 \\
0 \\
0\end{array}$ & & 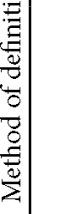 & $\mid \frac{\mathbb{Z}}{\widetilde{Z}}$ & 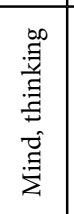 & 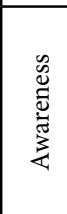 & & & & 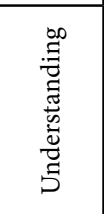 & 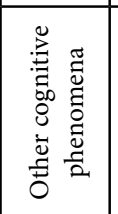 & 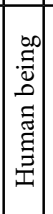 & 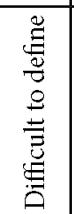 & | & \\
\hline$\stackrel{\Xi}{\stackrel{\Xi}{\Xi}}$ & t) & $\frac{9}{5}$ & $\therefore$ & $\stackrel{i}{i n}$ & ले & $\begin{array}{l}0 \\
\stackrel{+}{+} \\
\end{array}$ & $\underset{\tilde{i}}{\tilde{i}}$ & & ị & & & & & \\
\hline 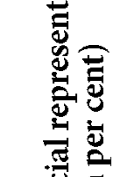 & 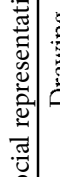 & 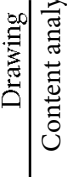 & 胥 & 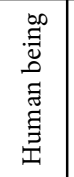 & 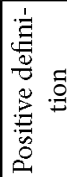 & 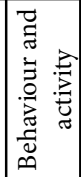 & 跑 & & 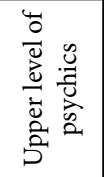 & & & & & \\
\hline 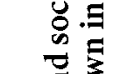 & के & & $\therefore$ & 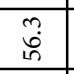 & 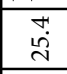 & 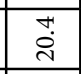 & & & $\begin{array}{l}\infty \\
\infty \\
\infty \\
-\infty \\
\end{array}$ & $\stackrel{\infty}{\stackrel{\infty}{\infty}}$ & \begin{tabular}{|l|}
$\infty$ \\
\\
\end{tabular} & $\stackrel{m}{\vec{g}}$ & & \\
\hline 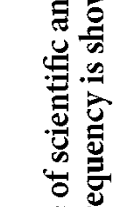 & & 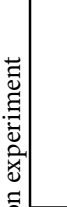 & $\frac{\pi}{\pi}$ & 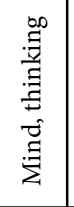 & 旅 & 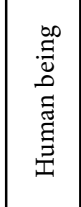 & & & 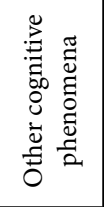 & 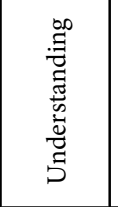 & 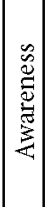 & $\begin{array}{l}\overline{0} \\
\overline{0}\end{array}$ & & \\
\hline 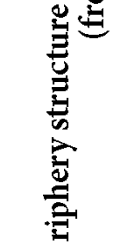 & & 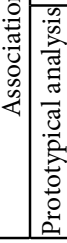 & $\stackrel{\mathbb{Z}}{=}$ & 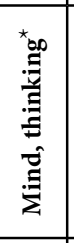 & $\mid$ & & & & 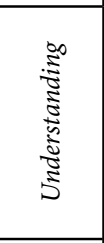 & 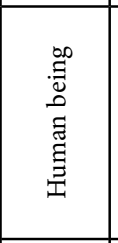 & 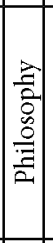 & 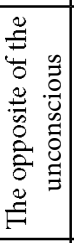 & 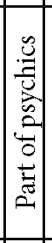 & 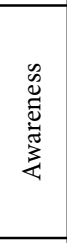 \\
\hline $\begin{array}{l}\vec{D} \\
\vec{d}\end{array}$ & : & $\cong$ & $\therefore$ & $\hat{m}$ & $\begin{array}{ll}+A \\
d\end{array}$ & & & & $\bar{\Xi}$ & $\vec{\Xi}$ & \begin{tabular}{|l|}
0 \\
\pm 0
\end{tabular} & $\begin{array}{l}0 \\
\pm \\
+10\end{array}$ & $\begin{array}{l}0 \\
\pm \\
\pm\end{array}$ & $\begin{array}{l}0 \\
\pm \\
f\end{array}$ \\
\hline $\begin{array}{l}\tilde{\delta} \\
\ddot{\Xi} \\
\ddot{E}\end{array}$ & 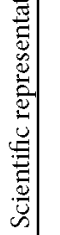 & 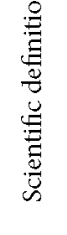 & $\mid$ & 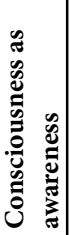 & 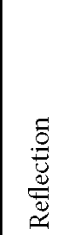 & & & & 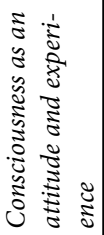 & 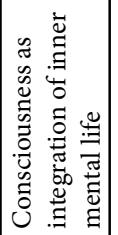 & 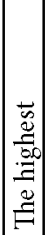 & 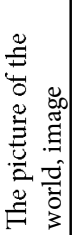 & 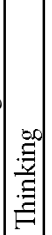 & 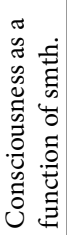 \\
\hline & & & & & & 407 & & & & $\theta^{r}$ & Н Т T & & LO & \\
\hline
\end{tabular}




\begin{tabular}{|c|c|c|c|c|c|c|c|c|c|c|}
\hline$\stackrel{m}{=}$ & $\begin{array}{l}\infty \\
\stackrel{\infty}{n} \\
\sigma\end{array}$ & $\begin{array}{l}\stackrel{n}{N} \\
\infty\end{array}$ & $\underset{\infty}{\infty}$ & $\stackrel{n}{n}$ & $\stackrel{n}{N}$ & 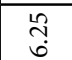 & If & & & \\
\hline 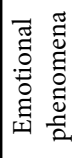 & 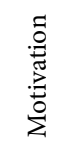 & 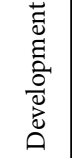 & 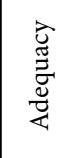 & 寻 & 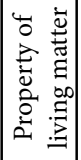 & 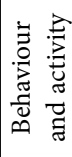 & 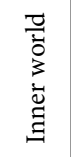 & & & \\
\hline$\sigma$ & $\begin{array}{l}0 \\
+\end{array}$ & & & & & & & & & \\
\hline 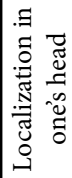 & 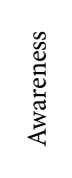 & & & & & & & & & \\
\hline 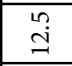 & 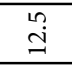 & $\overrightarrow{\mathrm{I}}$ & $\stackrel{\infty}{\infty}$ & $\begin{array}{l}+! \\
\stackrel{-}{\circ}\end{array}$ & $\begin{array}{l}\stackrel{\circ}{\circ} \\
\end{array}$ & $\begin{array}{l}19 \\
12 \\
\infty\end{array}$ & $\begin{array}{l}\infty \\
\stackrel{\Gamma}{\Lambda} \\
\end{array}$ & \begin{tabular}{|l|}
$\infty$ \\
0 \\
\end{tabular} & $\begin{array}{l}\hat{\hat{\sigma}} \\
\dot{b}\end{array}$ & $\begin{array}{l}\infty \\
\infty \\
i n \\
n\end{array}$ \\
\hline 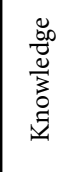 & 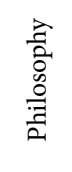 & 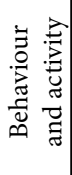 & 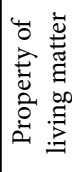 & 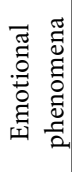 & 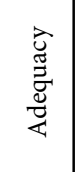 & 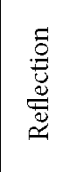 & 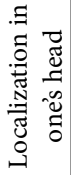 & 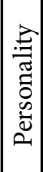 & 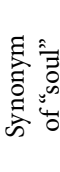 & 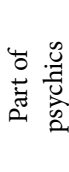 \\
\hline 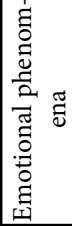 & 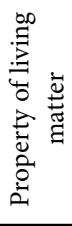 & 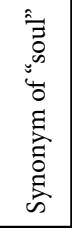 & 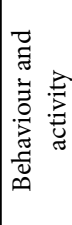 & 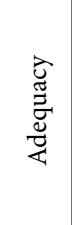 & 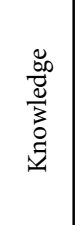 & & & & & \\
\hline Î & ָิ & $\stackrel{\sim}{N}$ & $\stackrel{2}{\sim}$ & $\stackrel{2}{n}$ & $\stackrel{N}{N}$ & $\stackrel{N}{N}$ & $\begin{array}{l}\infty \\
\infty \\
\infty \\
f\end{array}$ & \begin{tabular}{|l|}
$\infty$ \\
$\infty$ \\
$\gamma^{2}$
\end{tabular} & & \\
\hline 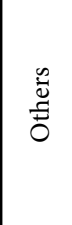 & 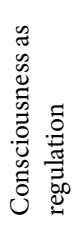 & 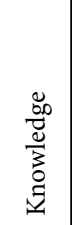 & 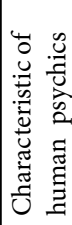 & 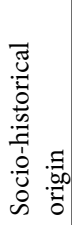 & 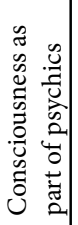 & 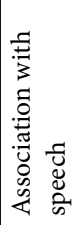 & 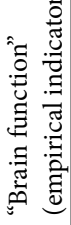 & 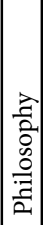 & & \\
\hline \multicolumn{11}{|c|}{ XצGHdI\&Gd } \\
\hline
\end{tabular}

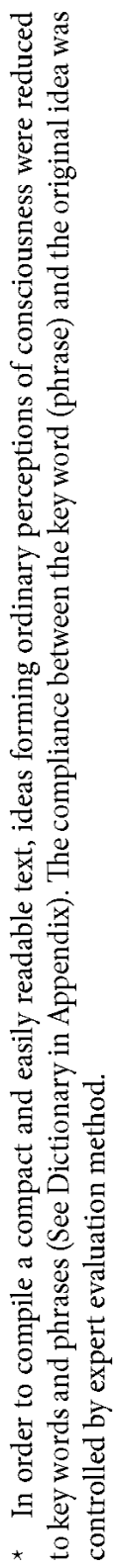


The resulting findings prove that the core of the scientific representations of consciousness is more compact and contains only two ideas: consciousness as awareness and consciousness as reflection. The core of social representations includes a larger number of ideas (nine), which are quite diverse. Both core ideas of the scientific representations are in a transformed way present in the core of social representations.

The area of potential changes of both scientific and social representations includes 6-7 ideas of each type, two of which are similar: consciousness as "the higher" (the top level of psychic evolution (Myasischev, 2001) and consciousness as the picture of the world. Two ideas from the potential change area of scientific representations - consciousness as the thinking and consciousness as a (brain) function - are present in the core of the social representations of consciousness. In other words, the frequency of the above ideas in collective consciousness is higher than that in scientific definitions.

The results of the comparison of the central core (Fig. 3) and the potential change area (Fig. 4) of scientific and social representations of consciousness are shown in Table 2.

Therefore, one can state that the potential area of changes of scientific representations contains a full range of ideas that have nothing in common with those contained in the core and the potential change area of social representations of consciousness. These ideas are: consciousness as an attitude and experience, and consciousness as an integration of inner mental life.

Besides, potential change area of social representations includes a number of ideas that are not present in the core and the potential change area of scientific representations. These ideas are: consciousness as an understanding of something, consciousness manifests itself in behaviour and activity, consciousness ensures human adequacy, consciousness receives a positive evaluation, consciousness as the opposite of the unconscious, and a difficulty in defining consciousness.

The analysis of ideas contained in the core and the potential change area of social representations of consciousness shows the dominance of the phenomena describing the learning activity of an individual, which supports the findings by M.S. Sheveleva (2003) and E.A. Shteinmets (2000).

The comparison of two types of representations makes it evident that scientific ideas are characterized with a higher abstractiveness, general- 


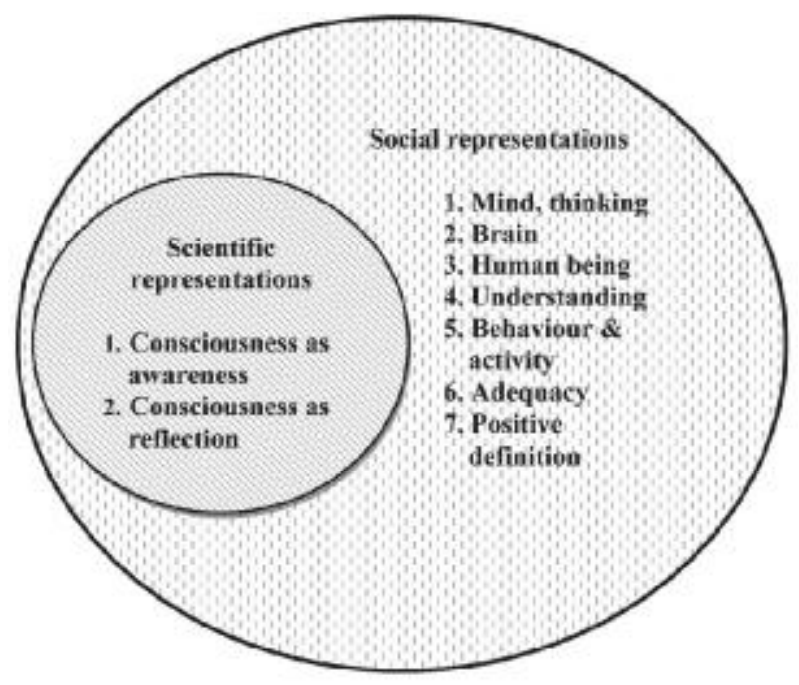

Figure 3. Graphic comparison of core areas of scientific and social representations of consciousness

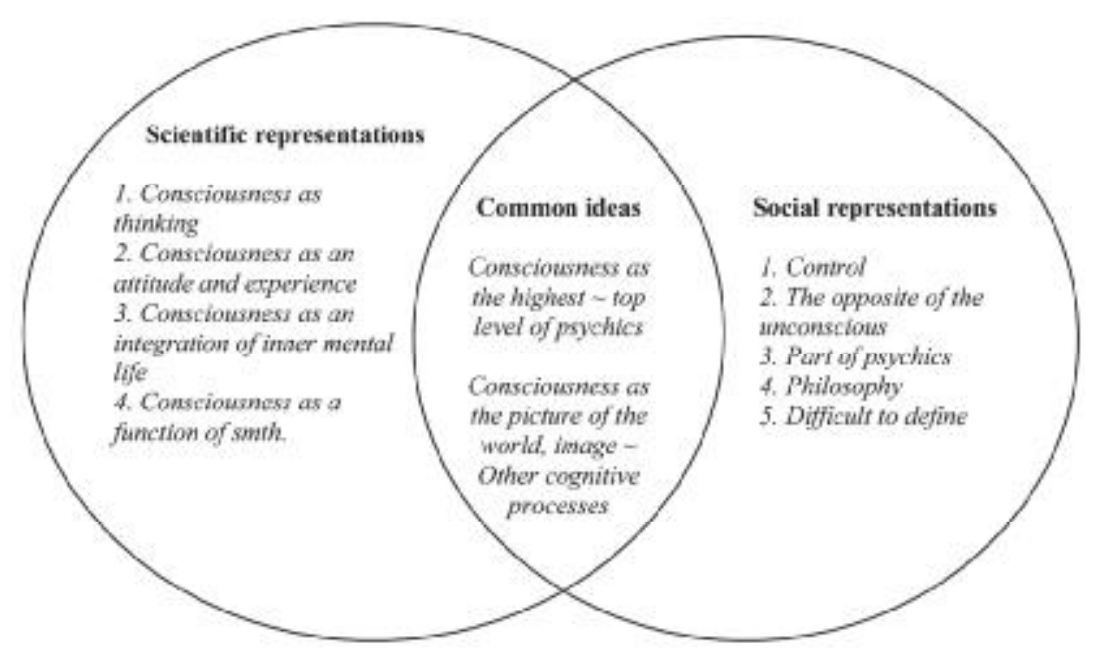

Figure 4. Graphic comparison of potential change areas of scientific and social representations of consciousness 


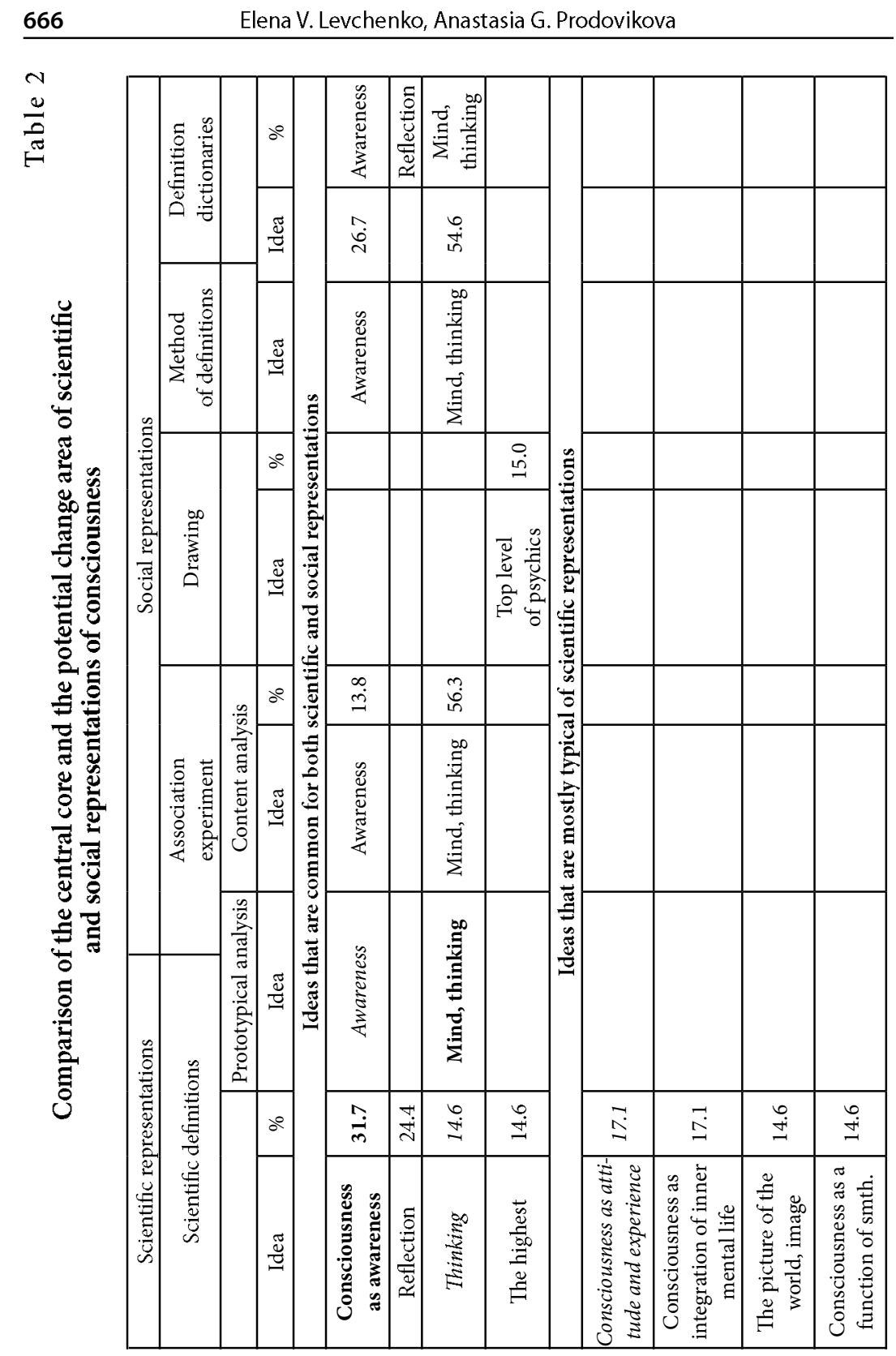




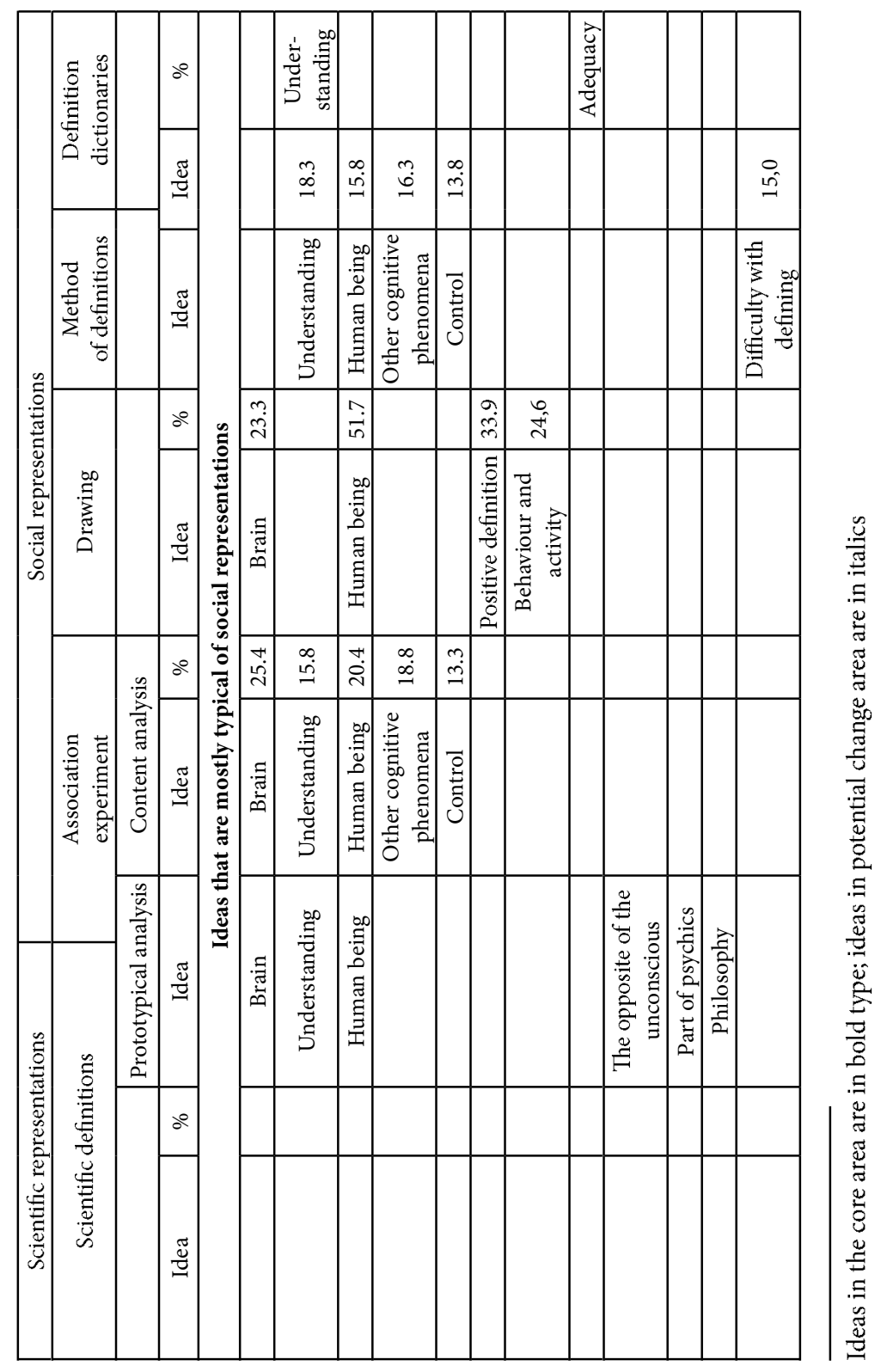




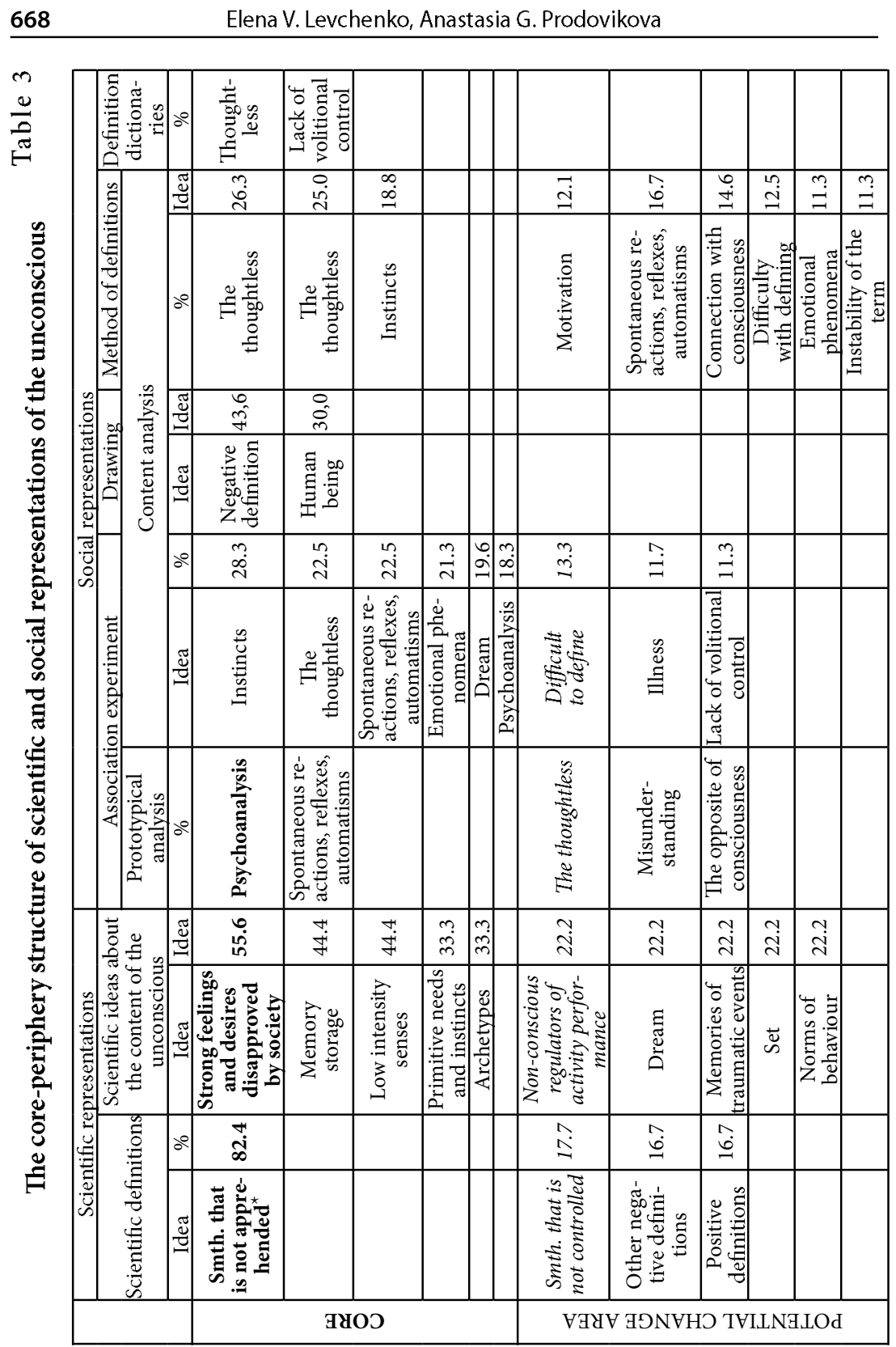




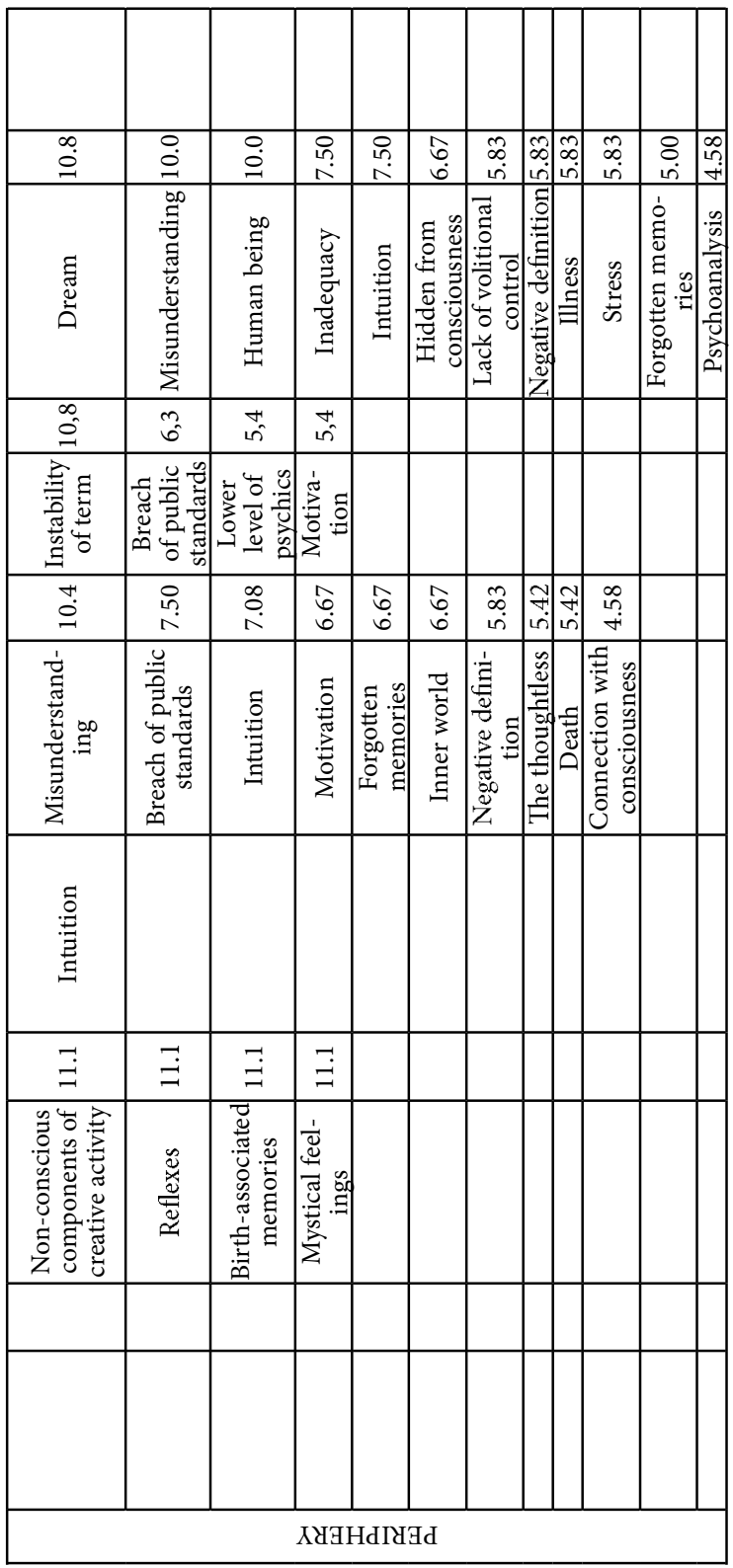

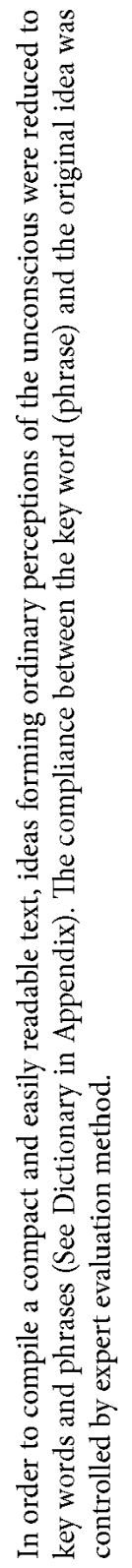




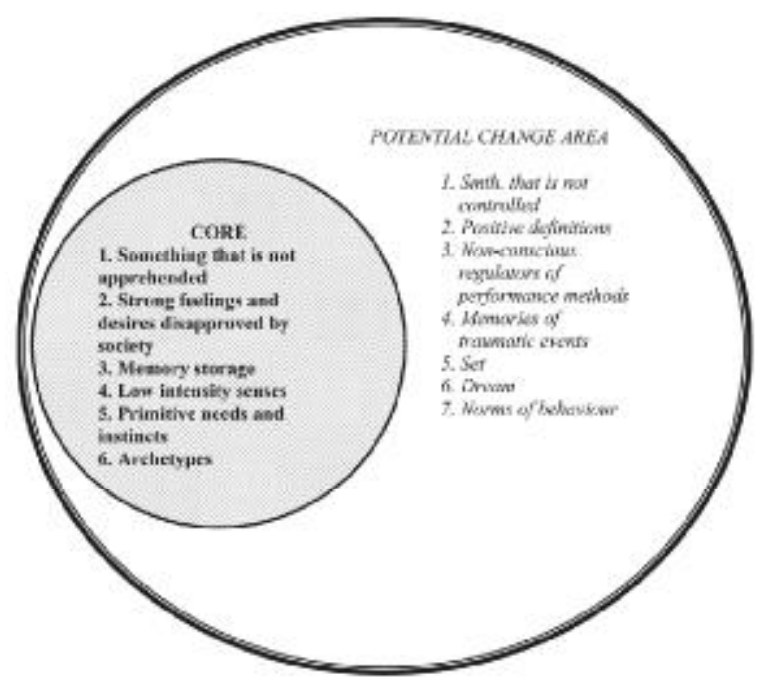

Figure 5. Scientific representations of the unconscious

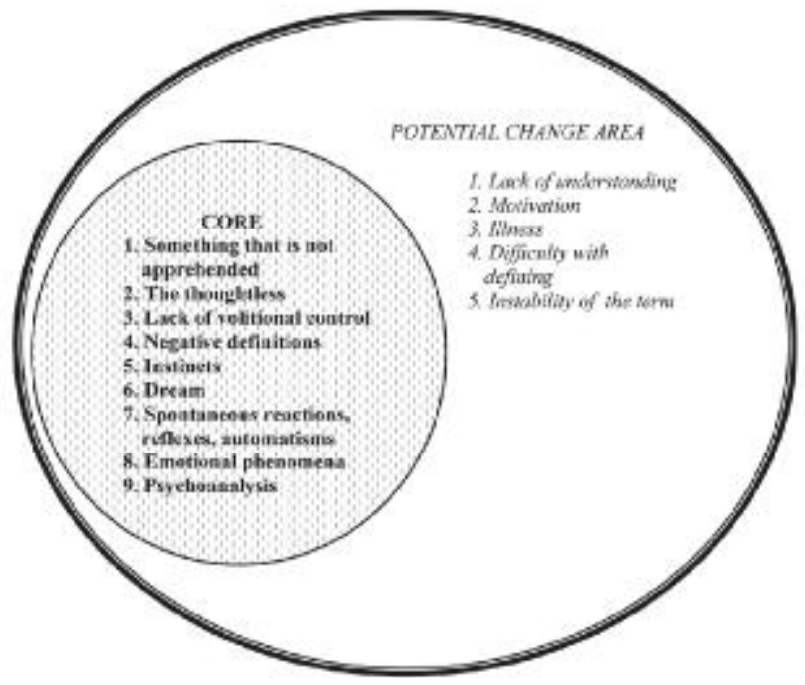

Figure 6. Social representations of the unconscious 
ity, accuracy of wording, and laconism, whereas social representations are characterized by concreteness, clarity, a larger freedom in the use of terms, and by the introduction of ideas originating from various areas of knowledge. The latter may be explained by the difference in functions between scientific and social representations. Scientific representations target at searching the essence, the truth. Social representations aim at helping the human being in his or her everyday life.

Let us turn to the representations of the unconscious. In a similar way, let us look at the core-periphery structures of scientific and social representations of the unconscious first in Table 3 and then in a graphic form.

Findings of the analysis reveal that the core of scientific representations of the unconscious contains fewer ideas and is more compact than the core of social representations. At the same time, one can find a number of ideas that are common for both scientific and the social representations of the unconscious. As follows from the Table and the Figures, the unconscious is defined in the negative way both in scientific and social representations. Notably, social representations describe the unconscious through the negation of ideas used in defining consciousness. The comparison of the central core and the potential change area of scientific and social representations of the unconscious is represented in Figure 7 and Table 4.

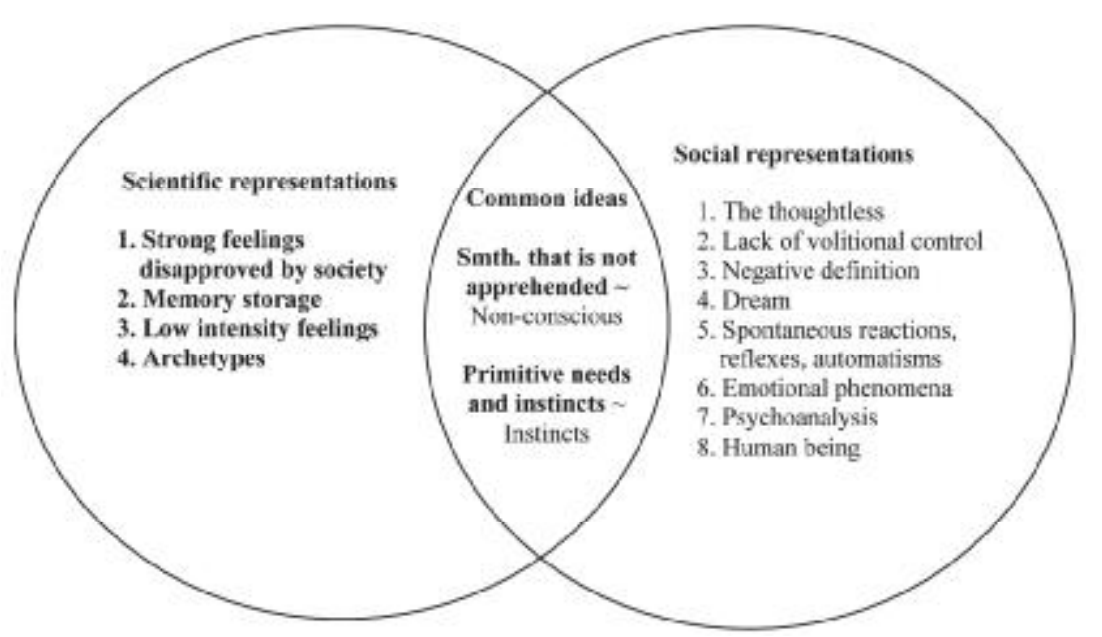

Figure 7. The graphic comparison of the core areas of scientific and social representations of the unconscious 


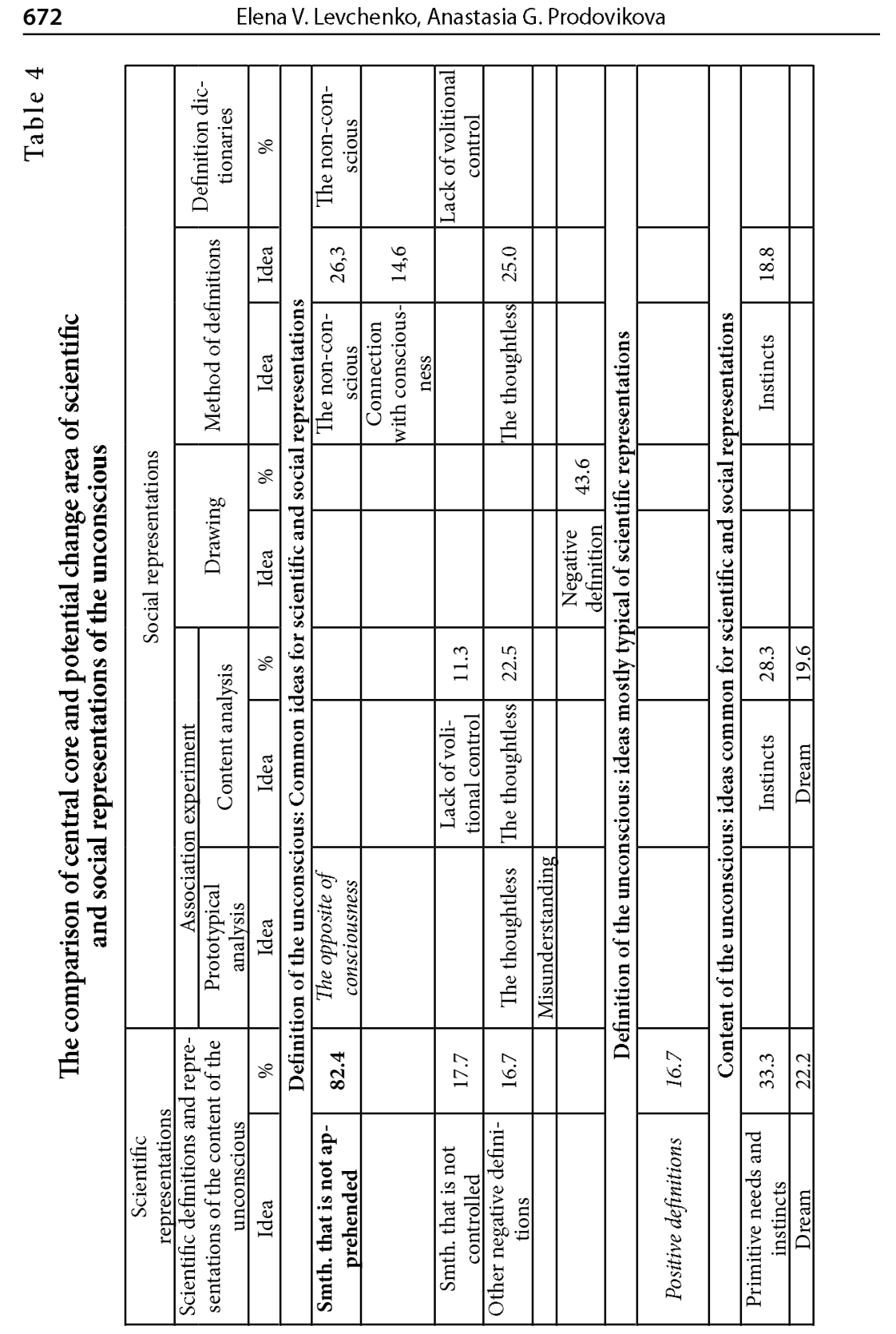


The Comparative Analysis of Scientific and Social Representations...

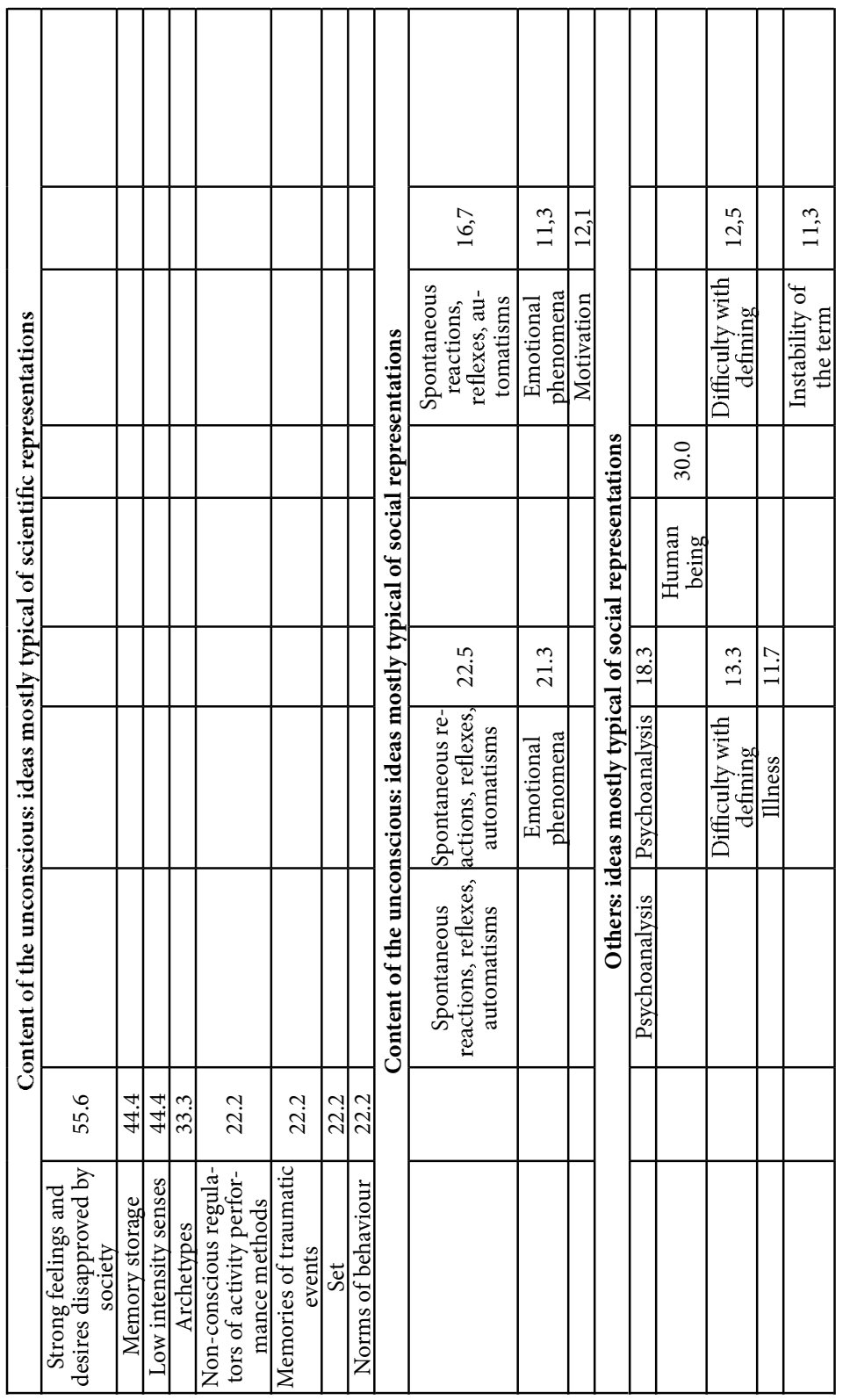


A comparison of the central core and the potential change area of scientific and social representations of the unconscious shows that ideas that are common for the core and the potential change area of scientific and social representations include instincts, dreams and automatisms in the sphere of the unconscious.

The idea of reflexes as elements of the sphere of the unconscious comes out of the periphery area of scientific representations of the unconscious and enters the core of social representations. The above fact proves that the level of "separability" of ideas (the term introduced by T.P. Emelyanova, 2006) in the group of scientists differs from that in the group of students.

The comparison also reveals the fact that the core of social representations of the unconscious does not contain any ideas that are not part of the core-periphery structure of scientific representations of the unconscious.

A number of ideas in the core and potential change area of social representations of the unconscious do not find support in collective consciousness. These include the idea that the unconscious may be defined in a positive way, as well as the ideas associated with various phenomena of the unconscious, such as memory storage, low intensity feelings, archetypes, memories of traumatic events, set, and norms of behaviour.

Social representations of the unconscious are characterized by the ideas describing various specific aspects of a phenomenon. This fact shows that social representations are more concrete. Analyzing the content of social representations of the unconscious one can note their orientation towards satisfying the everyday needs and wants of an individual.

Let us compare the obtained findings with the stages undergone by a scientific idea on the way of its transformation into social representations as they are described in literature. As seen in Table 5, social representations of consciousness show elements that indicate the second, the third and the forth stages of objectivation. The first stage of transformation took place quite a long time ago, and therefore the names of scientists who developed this concept are no longer associated with social representations. Social representations of the unconscious include elements referring to the first, second and third stages of objectivation. Speaking of the forth stage of the transformation of scientific ideas into social representations does not seem possible, as the word "the unconscious" itself has not been taken root in the Russian language yet and therefore cannot be regarded as a "substantial natural object." 
Table 5

Stages of transformation of scientific ideas into social representations

\begin{tabular}{|c|c|c|}
\hline Stages of objectivation & Ideas of consciousness & Idea of the unconscious \\
\hline $\begin{array}{l}\text { 1. Personification of } \\
\text { scientific knowledge } \\
\text { and phenomena. }\end{array}$ & $\begin{array}{l}\text { Although } 5 \% \text { of the subjects } \\
\text { mention the name of S. Freud } \\
\text { in association with the word } \\
\text { "consciousness," we believe } \\
\text { that this stage took place quite } \\
\text { a long time ago, and therefore } \\
\text { the names of scientists who } \\
\text { developed this concept are no } \\
\text { longer associated with social } \\
\text { representations. }\end{array}$ & $\begin{array}{l}\text { Manifests itself in mentioning the } \\
\text { names of S. Freud and C. Jung in the } \\
\text { findings of the association experi- } \\
\text { ment (13.75\%) and the method of } \\
\text { definitions. This is an evidence of } \\
\text { penetration of ideas of psychoanalysis } \\
\text { into collective consciousness. Let } \\
\text { us remember that in this country, } \\
\text { psychoanalysis was banned for a long } \\
\text { time. On the other hand, regardless } \\
\text { of the relatively frequent mentioning } \\
\text { of reflexes as part of the unconscious, } \\
\text { the name of I.P. Pavlov did not seem } \\
\text { to appear. Therefore, the idea of } \\
\text { reflexes has already grown into the } \\
\text { minds of nonprofessionals. }\end{array}$ \\
\hline $\begin{array}{l}\text { 2. Selection and exclu- } \\
\text { sion of separate ele- } \\
\text { ments of the scientific } \\
\text { theory (phenomenon) } \\
\text { from context. }\end{array}$ & \begin{tabular}{|l|} 
Not all scientific ideas are pre- \\
sented in collective conscious- \\
ness. Besides, some scientific \\
ideas have grown into social \\
representations, whereas \\
others are encountered rather \\
seldom.
\end{tabular} & $\begin{array}{l}\text { Only half of the scientific ideas } \\
\text { describing the content of the uncon- } \\
\text { scious are presented in collective con- } \\
\text { sciousness. Others have either failed } \\
\text { to penetrate collective consciousness } \\
\text { or are not regarded as useful in every- } \\
\text { day activities. Besides, some scientific } \\
\text { ideas have grown into social represen- } \\
\text { tations (e.g. "instincts"), while others } \\
\text { are encountered rater seldom (e.g. } \\
\text { "memory storage"). }\end{array}$ \\
\hline $\begin{array}{l}\text { 3. "Formation of a } \\
\text { figurative scheme," } \\
\text { "image core." }\end{array}$ & $\begin{array}{l}\text { The presence of figurative, } \\
\text { tangible elements of high fre- } \\
\text { quency: brain, human being, } \\
\text { upper world objects (i.e. sun, } \\
\text { sky, clouds, etc.) and others. }\end{array}$ & $\begin{array}{l}\text { The presence of figurative, tangible } \\
\text { elements of high frequency: lower } \\
\text { world objects (soil, darkness, demon, } \\
\text { etc.), human being, bottle (breach of } \\
\text { social code), bed, etc. }\end{array}$ \\
\hline $\begin{array}{l}\text { 4. "Naturalization," } \\
\text { "ontization" of the } \\
\text { "figurative scheme" } \\
\text { and its comprising ele- } \\
\text { ments, i.e. "attributing } \\
\text { material solidity to the } \\
\text { image." }\end{array}$ & $\begin{array}{l}\text { For instance, such phrases } \\
\text { as "our consciousness may } \\
\text { sometimes signal a coming } \\
\text { disaster," "consciousness may } \\
\text { control our thoughts and } \\
\text { acts," "consciousness inter- } \\
\text { nally controls the brain" show } \\
\text { that common sense operates } \\
\text { elements of the "figurative } \\
\text { scheme" as "substantial natu- } \\
\text { ral objects." }\end{array}$ & $\begin{array}{l}\text { Speaking of this stage does not seem } \\
\text { possible, as the word "the uncon- } \\
\text { scious" itself has not taken root in the } \\
\text { Russian language yet and therefore } \\
\text { cannot be regarded as a "substantial } \\
\text { natural object." }\end{array}$ \\
\hline
\end{tabular}



drawn:

The following conclusions based on the findings of our study can be

1. The core-periphery structure of scientific and social representations of consciousness and the unconscious that is typical of modern Russian culture and common to such carriers of this culture as active researchers in psychology and students has been reconstructed. All ideas forming the central core of scientific representations of consciousness penetrate in a transformed form in the core area of social representations. The central core of scientific and social representations of the unconscious is formed by a larger number of ideas than that of the representations of consciousness, which proves that representations of consciousness are characterized by a higher degree of stability than those of the unconscious.

2. Social representations of consciousness are first and foremost characterized by the phenomena describing the learning activity of an individual.

3. Representations of consciousness are an inexplicit basis for the formation of representations of the unconscious. Representations of the unconscious are constructed as negation of ideas expressed in representations of consciousness.

4. The core of social representations of consciousness and the unconscious include the ideas coming from the non-core area of scientific representations of these phenomena. In other words, the frequency of the same ideas in scientific and social representations varies.

5. The core area of social representations of consciousness contains a number of independent ideas that have not been part of scientific definitions associated with the phenomenon considered, which means that representations of consciousness has turned into an independent, autonomous entity, a phenomenon endowed with its own objective reality.

6. It has been demonstrated that the core of social representations of the unconscious does not include ideas that are not part of scientific representations. Therefore, scientific and social representations of the unconscious show a higher degree of similarity than those of consciousness.

7. Social representations of consciousness seem to include elements that are indicative of the forth stage of objectivation according to S. Moscovici. At the same time, social representations of the unconscious include elements only associated with the third stage of objectivation. 
8. As far as social representations of the unconscious are concerned, the potential change area, which plays an important role in the adaptation of representations to the changing context, includes a larger number of ideas borrowed from science and characterized by specific content than the potential change area of social representations of the unconscious. This proves higher stability of social representations of consciousness and their capacity to adapt to a new environment.

9. On the whole, the differences between scientific and social representations of consciousness and the unconscious derive from the fact that scientific representations have a more generalized and abstract nature and set stricter requirements to using terms and following the laws of logic. Social representations are characterized by greater concreteness, clarity, freedom in the use of terms, and inclusion of fragments from various fields of knowledge. The above mentioned differences between scientific and social representations are conditioned by the different functions of these representations. Scientific representations are aimed at seeking the essence of the phenomenon examined, seeking the objective truth, whereas social representations are meant to serve the "everyday" needs of an individual.

\section{References}

Abulkhanova, K.A. (2009). Soznanie kak žiznennaâ sposobnost' ličnosti [Consciousness as Life Ability of a Personality]. Psihologičeskij žurnal, 30 (1), 32-43.

Agafonov, A.Yu. (2003). Osnovy smyslovoj teorii soznaniâ [The Basics of the Semantic Theory of Consciousness]. Saint Petersburg: Reč.

Akopov, G.V. (2004). Problema soznaniâ v rossijskoj psihologii [The Problem of Consciousness in Russian Psychology]. Moscow, Voronezh: MPSI, NPO "MODÈK."

Akopov, G.V. (2007). Problema soznaniâ v sovremennoj psihologii: zarubežnye podhody [The Problem of Consciousness in Modern Psychology: Foreign Approaches]. In Psihologiâ soznaniâ: sovremennoe sostoânie i perspektivy: Materialy I Vserossijskoj konferencii: 29 iûnâ - 1 iûlâ 2007, Samara (pp. 77-89). Samara: Naučno-tehničeskij centr.

Allakhverdov, V.M. (2000). Soznanie kak paradoks [Consciousness as a Paradox]. Saint Petersburg: DNK.

Asmolov, A.G. (2002). Po tu storonu soznaniâ: metodologičeskie problemy neklassičeskoj psihologii [Beyond Consciousness: Methodological Problems of NonClassical Psychology]. Moscow: Smysl. 
Bekhterev, V.M. (1999). Psihika i žizn' [Psychics and Life]. Saint Petersburg: Aletejâ.

Bovina, I.B., \& Dragulskaya, L.Yu. (2006). Predstavleniâ studentov o nauke i ob učënom [Students' Representations of Science and a Scientist]. Voprosy psihologii, 6, 73-85.

Brushlinsky, A.V. (1998). Social'naâ psihologiâ v Rossii i teoriâ Serža Moskoviči [Social Psychology in Russia and Serge Moscovici's Theory]. In S. Moscovici, Vek tolp (preface). Moscow: Centr psihologii i psihoterapii.

Carroll, D.J., Apperly, I.A., \& Riggs, K.J. (2007). The Executive Demands of Strategic Reasoning are Modified by the Way in which Children are Prompted to Think about the Task: Evidence from 3- to 4-year-olds. Cognitive Development, 22 (1), 142-148.

Davydov, V.V., Lomov, B.F., Luria, A.R., Matyushkin, A.M., Petrovsky, A.V., Zaporozhets, A.V., \& Zinchenko, V.P. (Eds.). (1983). Psihologičeskij slovar' [The Psychological Dictionary]. Moscow: Pedagogika.

Emelyanova, T.P. (2001). Social'nye predstavleniâ - ponâtie i koncepciâ: itogi poslednego desâtiletiâ [Social Representations - the Concept and the Conception: The Results of the Past Decade]. Psihologičeskij žurnal, 6, 39-47.

Emelyanova, T.P. (2006). Konstruirovanie social'nyh predstavlenij v usloviâh transformacii rossijskogo obsestva [Constructing Social Representations in the Period of Transformation of Russian Society]. Moscow: IP RAN.

Freud, S. (2006). Osnovnye psihologičeskie teorii v psihoanalize [The Basic Psychological Theories in Psychoanalysis]. Moscow, Minsk: AST, Harvest.

Ganzen, V.A. (1984). Sistemnye opisaniâ v psihologii [Systemic Descriptions in Psychology]. Leningrad: LGU.

Grof, S. (2001). Putešestvie v poiskah sebâ [The Adventure of Self-Discovery]. Moscow: ITP, IPP.

James, W. (2001). Potok soznaniâ [The Stream of Consciousness]. In L.V. Kulikov (Ed.), Psihologiâ soznaniâ (pp. 8-19). Saint Petersburg: Piter.

Jaspers, K. (2001). Soznanie i bessoznatel'noe. Flûktuacii soznaniâ [Consciousness and the Unconscious. Fluctuations of Consciousness]. In L.V. Kulikov (Ed.), Psihologiâ soznaniâ (pp. 225-241). Saint Petersburg: Piter.

Jung, C.G. (1994). Psihologiâ bessoznatel'nogo [Psychology of the Unconsciousness]. Moscow: Kanon.

Kruglanski, A.W. (2007). Implicit Theory of Personality as a Theory of Personality. In A.W. Kruglanski \& E.T. Higgins (Eds.), Social Psychology: handbook of Basic Principles. 2nd ed. New York: The Guilford Press.

Leibniz, G.W. (1982). Sočineniâ v četyreh tomah: T. I. [Selected Works in four volumes. Vol. 1]. Moscow: Mysl'.

Leontiev, A.N. (1975). Deâtel'nost'. Soznanie. Ličnost' [Activity. Consciousness. Personality]. Moscow: Politizdat. 
Levchenko, E.V. (2008). Psihoanaliz i psihologičeskoe znanie: dinamika otobraženij psihoanaliza $\mathrm{v}$ otečestvennyh učebnikah psihologii [Psychoanalysis and Psychological Knowledge: The Dynamic of Reflections of Psychoanalysis in Russian Psychology Textbooks]. In E.V. Levchenko (Ed.), Psihologiâ buduŝego: materialy mežvuzovskoj naučnopraktičeskoj konferencii, posvâŝennoj 150-letiû so dnâ roždeniâ Z. Frejda (pp. 3-21). Perm.

Lomov, B.F. (1984). Metodologičeskie i teoretičeskie problemy psihologii [Methodological and Theoretical Problems of Psychology]. Moscow: Nauka.

Luria, A.R. (1979). Âzyk i soznanie [Language and Consciousness]. Moscow: MGU.

Meshcheriakov, B.G., \& Zinchenko, V.P. (Eds.). (2003). Bol'šoj psihologičeskij slovar' [The Big Psychological Dictionary]. Saint Petersburg: prajm-EVROZNAK.

Moscovici, S. (1961). La Psychanalyse, son Image et son Public. Paris: PUF.

Myasischev, V.N. (2001). Soznanie kak edinstvo otraženiâ dejstvitel’nosti i otnošenij $\mathrm{k}$ nej čeloveka [Consciousness as a Unity of Reflection of Reality and Human Attitude to it]. In L.V. Kulikov (Ed.), Psihologiâ soznaniâ (pp. 56-63). Saint Petersburg: Piter.

Ozhegov, S.I. (1989). Slovar' russkogo âzyka [The Dictionary of the Russian Language]. Moscow: Russkij âzyk.

Parkhomenko, O.G. (2003). Implicitnye teorii intellekta studentov pedagogičeskogo universiteta [Implicit Theories of Intelligence of Pedagogical University Students] (Candidate of Sciences dissertation). Moscow.

Platonov, K.K. (1972). O sisteme psihologii [On the System of Psychology]. Moscow: Mysl'.

Podprugina, V.V. (2003). Mental'nye reprezentacii bazovyh èmocij [Mental Representations of Basic Emotions] (Candidate of Sciences dissertation). Moscow.

Reber, A. (2000). Bol'soj tolkovyj psihologičeskij slovar'. T. 2 (P - Â) [The Penguin Dictionary of Psychology]. Moscow: Veče, AST.

Rubinstein, S.L. (1957). Bytie i soznanie. O meste psihičeskogo vo vseobŝej vzaimosvâzi âvlenij material'nogo mira [Being and Consciousness. On the Place of the Psychic in the General Interconnection of the Material World Phenomena]. Moscow: AN SSSP.

Savina, E.A. (1995). Osobennosti predstavlenij detej 5-10 let o duše [The Peculiarities of 5-10 Years Old Children's Notions of a Soul]. Voprosy psihologii, 3, 21-28.

Searle, J.R. (1980). Minds, Brains, and Programs. Behavioral and Brain Sciences, 3, 417-457.

Shevchenko, O.Yu. (2003). Učebno-professional'nầ ustanovka $v$ strukture professional'nogo soznaniâ studentov vuzov [The Educational and Occupational Set in the Context of Students' Professional Consciousness] (Candidate of Sciences dissertation). Moscow.

Sheveleva, M.S. (2003). Psihika i âvleniâ psihiki: Predstavlennost'v myšlenii [The Psychics and the Psychic Phenomena] (Candidate of Sciences dissertation). Moscow. 
Shikhirev, P.N. (1999). Sovremennaâ social'naâ psihologiâ [Modern Social Psychology]. Moscow: IP RAN, KSP+, Akademičeskij Proekt.

Shteinmets, A.E. (2000). Donaučnye predstavleniâ studentov-psihologov v kontekste prepodavaniâ psihologii [Pre-Scientific Representations of Psychology Students in the Context of Teaching Psychology]. Voprosy psihologii, 4, 124-132.

Shvedova, N.Yu. (Ed.). (2007). Tolkovyj slovar' russkogo âzyka s vklûčeniem svedenij o proishoždenii slov [The Russian Definition Dictionary supplied with Etymological Data]. Moscow: IRÂ RAN.

Simonov, P.V. (2001). O dvuh raznovidnostâh neosoznavaemogo psihičeskogo: pod- i sverhsoznanii [On the Two Varieties of the Non-Conscious Psychic: The Subconscious and the Super-Conscious]. In L.V. Kulikov (Ed.), Psihologiâ soznaniâ (pp. 137-148). Saint Petersburg: Piter.

Sitnikov, V.L. (2003). Psihologiâa obraza rebenka v soznanii sub"ektovpedagogičeskogo processa [The Psychology of the Image of a Child in the Consciousness of the Subjects of the Pedagogical Process] (Doctor of Sciences dissertation). Moscow.

Sobel, D.M. (2007). Children's Knowledge of the Relation between Intentional Action and Pretending. Cognitive Development, 22 (1), 130-141.

Subbotsky, E.V. (1985). Predstavlenie rebenka o sootnošenii telesnyh i psihičeskih âvlenij [A Child's Notion of the Correlation of Bodily and Psychic Phenomena]. Vestnik Moskovskogo universiteta. Seriâ 14 "Psihologiâ," 2, 38-50.

Subbotsky, E.V. (1999). Individual'noe soznanie kak sistema real'nostej [Individual Consciousness as a System of Realities]. In A.E. Voiskounsky, A.N. Zhdan, \& O.K. Tikhomirov (Eds.), Tradicii i perspektivy deâtel'nostnogo podhoda $v$ psihologii: škola A.N. Leont'eva (pp. 114-145). Moscow: Smysl.

Uznadze, D.N. (1966). Psihologičeskie issledovaniâ [Psychological Studies (selected works)]. Moscow: Nauka.

Vakhrusheva, A.S. (2003). Verbal'noe oposredovanie v stanovlenii predmetnogo soznaniâ [Verbal Mediation in the Development of Object Consciousness] (Candidate of Sciences dissertation). Barnaul.

Vasiluk, F.E. (2007). Ponimaûsââ psihoterapiâ kak psihotehničeskaâ sistema ["Understanding Psychotherapy" as a Psychotechnical System] (Doctor of Sciences dissertation). Moscow.

Wundt, W. (2002). Vvedenie v psihologiû [Introduction to Psychology]. Saint Petersburg: Piter.

Zhdan, A.N. (2002). Istoriâ psihologii: ot antičnosti k sovremennosti [The History of Psychology: From Antiquity to the Present Day]. Moscow: Pedagogičeskoe obŝestvo Rossii.

Zinchenko, V.P. (2001). Miry soznaniâ i struktura soznaniâ [The Worlds of Consciousness and the Structure of Consciousness]. In L.V. Kulikov (Ed.), Psihologiâ soznaniâ (pp. 149-162). Saint Petersburg: Piter. 


\section{Appendix: Glossary}

In order to make the text compact, the ideas forming social representations of consciousness and the unconscious have been reduced to key words and phrases. The degree of correspondence between the key word (phrase) and the original idea has been controlled by the expert evaluation method. For the reader's convenience, the following glossary has been compiled; it contains the list of the key concepts and reveals the ideas corresponding to them in the examined texts.

Table 6

The key words and ideas forming social representations of consciousness

\begin{tabular}{|c|c|}
\hline Key word (phrase) & Ideas \\
\hline Adequacy & $\begin{array}{l}\text { 1. Adequacy of behavior in a human being in a conscious state. } \\
\text { 2. It ensures adequacy of perception and behavior. } \\
\text { 3. "A condition of an individual in healthy mind and memory, an ability } \\
\text { to be aware of one's behaviour and feelings" (Ozhegov, 1989). }\end{array}$ \\
\hline Awareness & $\begin{array}{l}\text { 1. Consciousness as awareness of one's "ego," self-consciousness is part } \\
\text { of the structure of consciousness. } \\
\text { 2. Thanks to consciousness, one can be aware of oneself, one's actions } \\
\text { and the outside world. } \\
\text { 3. See "realize," "confess": same as to be aware of (Ozhegov, 1989). }\end{array}$ \\
\hline Brain & $\begin{array}{l}\text { 1. Activity of consciousness (mind) is ensured by the brain activity. } \\
\text { 2. The nervous system and the brain are the material foundation of } \\
\text { activity of consciousness. }\end{array}$ \\
\hline Behaviour and activity & $\begin{array}{l}\text { 1. Consciousness manifests itself through an individual's actions. } \\
\text { 2. Consciousness manifests itself in behavior and activity. } \\
\text { 3. Thanks to human consciousness, a world of culture emerges. }\end{array}$ \\
\hline Control & It enables a human being to control his or her behavior. \\
\hline Difficulty with defining & The concept of "consciousness" is hard to define. \\
\hline Emotional phenomena & $\begin{array}{l}\text { 1. Apart from the rational component, consciousness also includes ir- } \\
\text { rational components, i.e. feelings, senses, sensations. } \\
\text { 2. Consciousness includes phenomena of the emotional sphere. }\end{array}$ \\
\hline Development & Consciousness is subject to development. \\
\hline Human being & $\begin{array}{l}\text { 1. The carrier of consciousness is a human being. } \\
\text { 2. The carrier of mind is a human being. }\end{array}$ \\
\hline Inner world & Consciousness may be regarded as the inner world of a human being. \\
\hline Knowledge & $\begin{array}{l}\text { 1. Knowledge as a result of the activity of mind. } \\
\text { 2. Consciousness ensures learning activity the product of which is } \\
\text { knowledge. }\end{array}$ \\
\hline $\begin{array}{l}\text { Localization in one's } \\
\text { head }\end{array}$ & Consciousness is located in one's head. \\
\hline
\end{tabular}




\begin{tabular}{|c|c|}
\hline Mind, thinking & $\begin{array}{l}\text { 1. Consciousness is mind (intelligence). } \\
\text { 2. Mind ensures the psychic process of thinking resulting in thoughts. } \\
\text { 3. Consciousness is the same as mind, intelligence. } \\
\text { 4. The same as conscientiousness: an ability to properly understand the } \\
\text { surrounding reality, identify one's behavior (Ozhegov, 1989). }\end{array}$ \\
\hline Motivation & Consciousness performs a driving function. \\
\hline $\begin{array}{l}\text { Other cognitive phe- } \\
\text { nomena }\end{array}$ & It also includes other phenomena of the cognitive sphere. \\
\hline Part of psychics & $\begin{array}{l}\text { 1. Consciousness is a directly given part of psychics. } \\
\text { 2. Consciousness is the only directly observed part of psychics; con- } \\
\text { sciousness is an object of psychological research. }\end{array}$ \\
\hline Personality & It determines an individual's personality. \\
\hline Philosophy & $\begin{array}{l}\text { 1. Philosophical understanding of consciousness as the antithesis of } \\
\text { matter. } \\
\text { 2. Consciousness is an object of study in philosophy. }\end{array}$ \\
\hline Positive definition & The word "consciousness" receives a positive definition. \\
\hline $\begin{array}{l}\text { Property of living } \\
\text { matter }\end{array}$ & $\begin{array}{l}\text { 1. Consciousness is the property of a living body; it is associated with } \\
\text { life. } \\
\text { 2. Consciousness is a property of a human being. }\end{array}$ \\
\hline Reflection & $\begin{array}{l}\text { 1. Consciousness reflects the outside world. } \\
\text { 2. It is a "human ability to reproduce reality in thinking; mental activity } \\
\text { as reflection of reality" (Ozhegov, 1989). }\end{array}$ \\
\hline $\begin{array}{l}\text { Synonym to the word } \\
\text { "soul" }\end{array}$ & $\begin{array}{l}\text { 1. Consciousness as similar in meaning to the word "soul." } \\
\text { 2. Consciousness and soul may sometimes be used as synonyms. }\end{array}$ \\
\hline $\begin{array}{l}\text { The opposite of the } \\
\text { unconscious }\end{array}$ & Consciousness is opposed to the subconscious, the unconscious. \\
\hline Understanding & $\begin{array}{l}\text { 1. The activity of mind may also result in understanding something. } \\
\text { 2. The activity of consciousness results in understanding something. } \\
\text { 3. "A thought, a feeling, understanding something clearly" (Ozhegov, } \\
\text { 1989). }\end{array}$ \\
\hline $\begin{array}{l}\text { Upper level of the } \\
\text { psychics }\end{array}$ & Consciousness is the upper level of the psychics. \\
\hline
\end{tabular}

Table 7

The key words and ideas forming social representations of the unconscious

\begin{tabular}{|l|l|}
\hline \multicolumn{1}{|c|}{ Key word (phrase) } & \multicolumn{1}{c|}{ Ideas } \\
\hline $\begin{array}{l}\text { Breach of public } \\
\text { standards }\end{array}$ & It manifests itself in the breach of rules accepted in society. \\
\hline $\begin{array}{l}\text { Connection with } \\
\text { consciousness }\end{array}$ & $\begin{array}{l}\text { The unconscious is defined in connection with the concept of "con- } \\
\text { sciousness": either as its "continuation" or as its opposite. }\end{array}$ \\
\hline Death & The dead person is unconscious. \\
\hline
\end{tabular}


The Comparative Analysis of Scientific and Social Representations...

\begin{tabular}{|c|c|}
\hline Difficulty with defining & The phenomenon of the unconscious is hard to define and explain. \\
\hline Dream & The unconscious is reflected in dreams. \\
\hline Emotional phenomena & It includes emotions and feelings. \\
\hline Forgotten memories & It contains forgotten memories. \\
\hline $\begin{array}{l}\text { Something hidden } \\
\text { from consciousness }\end{array}$ & The unconscious is hidden from consciousness. \\
\hline Human being & The carrier of the unconscious is a human being. \\
\hline Illness & The unconscious is inherent to illness. \\
\hline Inadequacy & $\begin{array}{l}\text { The unconscious state is characterized by the inadequacy of percep- } \\
\text { tion, thoughts, behavior. }\end{array}$ \\
\hline Inner world & The unconscious is part of the inner world. \\
\hline Instability of term & $\begin{array}{l}\text { The term "the unconscious" has not taken root in the Russian lan- } \\
\text { guage. }\end{array}$ \\
\hline Instincts & The unconscious includes animal instincts. \\
\hline Intuition & $\begin{array}{l}\text { 1. Intuition as part of the unconscious is a way of learning. } \\
\text { 2. The unconscious manifests itself through the phenomenon of } \\
\text { intuition. }\end{array}$ \\
\hline $\begin{array}{l}\text { Lack of volitional } \\
\text { control }\end{array}$ & $\begin{array}{l}\text { 1. The unconscious condition lacks volitional control. } \\
\text { 2. "Unconscious" is instinctive, unaccountable (Ozhegov, 1989). }\end{array}$ \\
\hline Lower level of psychics & The unconscious is the lower level of psychics. \\
\hline Misunderstanding & $\begin{array}{l}\text { 1. The unconscious and its manifestations are misunderstood by } \\
\text { respondents. } \\
\text { 2. Unconscious behavior is beyond one's understanding. }\end{array}$ \\
\hline Motivation & It is a driving force of human behavior. \\
\hline Negative definition & The unconscious receives a negative definition. \\
\hline Psychoanalysis & $\begin{array}{l}\text { 1. S. Freud is the founder of psychoanalysis, a school that regards the } \\
\text { unconscious as the central phenomenon of human psychics. The con- } \\
\text { cepts of "dream," "instinct," the "subconscious" constitute significant } \\
\text { elements of the psychoanalytical conception. } \\
\text { 2. The unconscious is an object of psychoanalytical study. }\end{array}$ \\
\hline $\begin{array}{l}\text { Something that is non- } \\
\text { conscious }\end{array}$ & $\begin{array}{l}\text { 1. The unconscious is something that is non-conscious. } \\
\text { 2. Accompanied by the loss of consciousness (Ozhegov, 1989). }\end{array}$ \\
\hline $\begin{array}{l}\text { Spontaneous reactions, } \\
\text { reflexes and automa- } \\
\text { tisms }\end{array}$ & $\begin{array}{l}\text { 1. The unconscious includes reflexes. } \\
\text { 2. Unconscious behavior cannot be controlled by consciousness, it is } \\
\text { reflected in spontaneous reactions, reflexes, and automatisms. }\end{array}$ \\
\hline Stress & The unconscious reveals itself under stressful circumstances. \\
\hline $\begin{array}{l}\text { The opposite of con- } \\
\text { sciousness }\end{array}$ & Consciousness is opposed to the unconscious. \\
\hline Thoughtless & $\begin{array}{l}\text { 1. The unconscious is associated with thoughtless, irrational behaviors } \\
\text { and actions. } \\
\text { 2. It is characterized by the lack of logic manifested in thoughtless, } \\
\text { irrational behavior. }\end{array}$ \\
\hline
\end{tabular}

